\title{
Computational Investigation of Pro-arrhythmogenesis of Heart Failure Induced Electrical Remodelling in Ventricles
}

\author{
Kun Jian, Chen Li, Gareth Jones, Henggui Zhang \\ University of Manchester, Manchester, UK
}

\begin{abstract}
Heart failure (HF) is one of the most common cardiac diseases that are associated with increased susceptibility of ventricular arrhythmia causing morbidity and mortality. Previous studies have found that HF causes a series of electrical and structural remodeling in the heart. The aim of this study was to determine the predominant factor of HF-induced remodeling responsible for ventricular arrhythmias.

We developed a new family of cellular electrical models of canine ventricular and Purkinje fiber (PF) myocytes in control and HF condition based on experimental data. These models were validated by reproducing experimentally observed AP prolongation in HF condition. Single cell models were then incorporated into a $3 D$ anatomical model with detailed electrical heterogeneity and structural anisotropy, as well as PF network. Re-entrant excitation waves were initiated using the phase distribution method. Role of each individual HF-induced electrical and structural remodeling on lifespan of re-entry was investigated.

Among HF-induced remodeling, the reduction of intercellular conductivity plays an important role in sustaining ventricular arrhythmia. The ion channel remodeling though prolongs APD leading to increased vulnerability, it however reduces sustainability of reentry.
\end{abstract}

\section{Introduction}

According to the reports, there are estimated to be 14.9 million patients across the EU [1] and 5.7 million in the US living with HF [2].25\% of HF patients die within one year of diagnosis [3] and 50\% die within five years [4]. Sudden death normally caused by cardiac arrhythmias accounts for up to $50 \%$ of deaths among patients with HF.

Previous studies have found that HF causes a series of electrical and structural remodelling in the heart, which may be responsible for arrhythmogenesis. Firstly, action potential duration (APD) prolongation is the most commonly observed feature in animal HF models.
Secondly, connexin43, which reflects intercellular electrical coupling, can be reduced by up to $50 \%$ in $\mathrm{HF}$ conditions. Thirdly, in the case of HF induced by dilated cardiomyopathy (DCM), the ventricle is dilated and has a thinner wall thickness and an enlarged chamber volume.

In this study, we developed a biophysically detailed 3D model of canine ventricles. Using the model, we investigated the pro-arrhythmic effects of HF-induced remodelling. The role of each individual HF-induced electrical and structural remodelling on the lifespan of reentrant excitation waves is investigated.
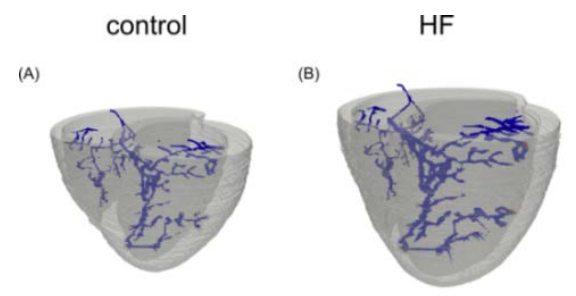

(C)

(D)
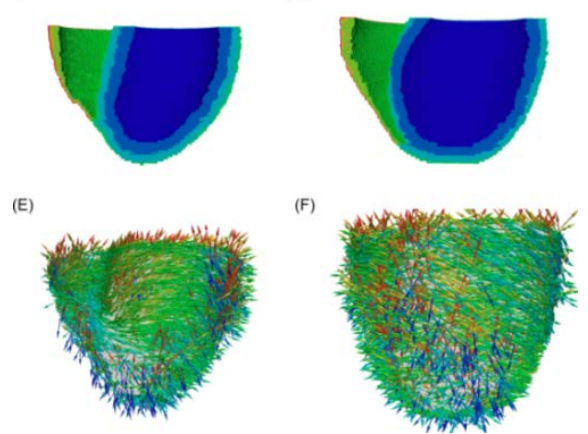

Figure 1. Anatomical geometries of the normal and the failing ventricles. A-B: Incorporation of the His-Purkinje system in to the ventricles. C-D: Segmentation of the ventricular wall. E-F: Map of fiber orientations.

\section{Methods}

\subsection{Single cell simulations}

In order to determine the predominant factor of the HF-induced remodelling that responsible for ventricular arrhythmias, we developed two families of canine single 
cell models: one is for canine ventricular cells, which was developed by modifying the Benson et al. [5] models. These single cell models take into account of the transmural heterogeneity of the ventricle wall, forming three different ventricular cell types, i.e., the endocardium, the mid-myocardium and the epicardium cell. The other is the canine Purkinje Fibre cell model which was developed by modifying the Aslanidi et al. [6] model.

HF-induced ionic remodelling were simulated based on comprehensive experimental data. These modifications include the update of the original Benson et al. [5] and Aslanidi et al. [6] models as well as incorporation of the HF-induced ionic channel remodelling on $\mathrm{I}_{\mathrm{Na}}, \mathrm{I}_{\mathrm{K} 1}, \mathrm{I}_{\mathrm{to} 1}, \mathrm{I}_{\mathrm{Kr}}, \mathrm{I}_{\mathrm{Ks}}, \mathrm{I}_{\mathrm{CaL}}, \mathrm{I}_{\mathrm{CaT}},\left[\mathrm{Ca}^{2+}\right]_{\mathrm{i}}, \mathrm{I}_{\mathrm{NaK}}$ and $\mathrm{Na}^{+}-\mathrm{Ca}^{2+}$ exchange current (NCX).

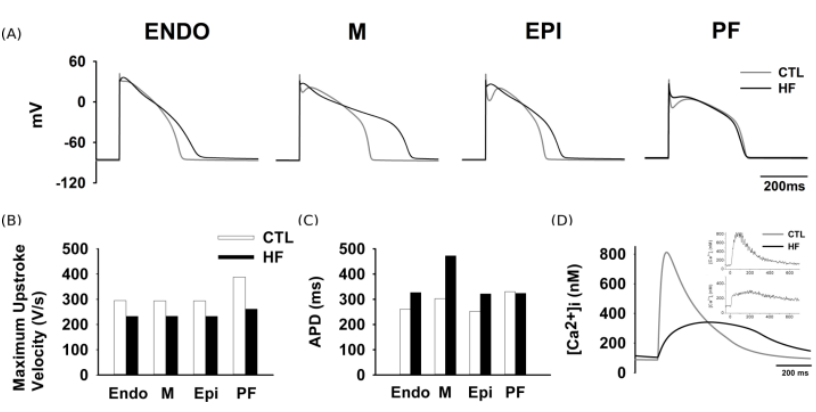

Figure 2. Time courses of action potentials (A), maximum upstroke velocity (B), APD (C) and intracellular calcium with experimental data [7] shown in insets (D).

\subsection{Anatomical geometries manipulation}

Two anatomical geometries were implemented which were downloaded from the CardioVascular Research Grid (CVRG): one for normal canine ventricles and the other for failing canine ventricles that were reconstructed based on the DT-MRI data [7-9]. Both ventricles were then interpolated and segmented into the endocardium, the mid-myocardium and the epicardium with a constitutional portion of $60 \%$, 30\% and $10 \%$ respectively [11]. The HisPurkinje network created by Panfilov [12] was rescaled and incorporated into the ventricles.

\subsection{D electrophysiological simulations}

Action potential propagation is described using the monodomain equation in this study:

$$
\frac{\partial V_{m}}{\partial t}=\nabla \cdot \boldsymbol{D}\left(\nabla V_{m}\right)-\frac{I_{i o n}}{C_{m}}
$$

where $V_{m}$ is the membrane potential, $\nabla$ is the $3 \mathrm{D}$ spatial Laplacian defined within the tissue, $C_{m}$ is the cell capacitance per unit surface area, $I_{\text {ion }}$ is the sum of all transmembrane ionic currents and $\boldsymbol{D}$ is the tensor of diffusion coefficient given by:

$$
\boldsymbol{D}=\boldsymbol{f} \mathrm{D}_{\|} \boldsymbol{f}^{\mathrm{T}}+\boldsymbol{s} \mathrm{D}_{\perp 1} \boldsymbol{s}^{\mathrm{T}}+\boldsymbol{n} \mathrm{D}_{\perp 2} \boldsymbol{n}^{\mathrm{T}}
$$

where $\boldsymbol{f}, \boldsymbol{s}$ and $\boldsymbol{n}$ represent the vectors in the direction along fibre, in the direction perpendicular to fibre in sheet, and in the direction perpendicular to sheet respectively. $D_{\|}, D_{\perp 1}$ and $D_{\perp 2}$ represent the diffusion coefficients in these directions. The diffusion coefficients were determined based on experimental data [13] and resulted in $40 \%$ reduction of the electrical conduction velocity in the HF condition.

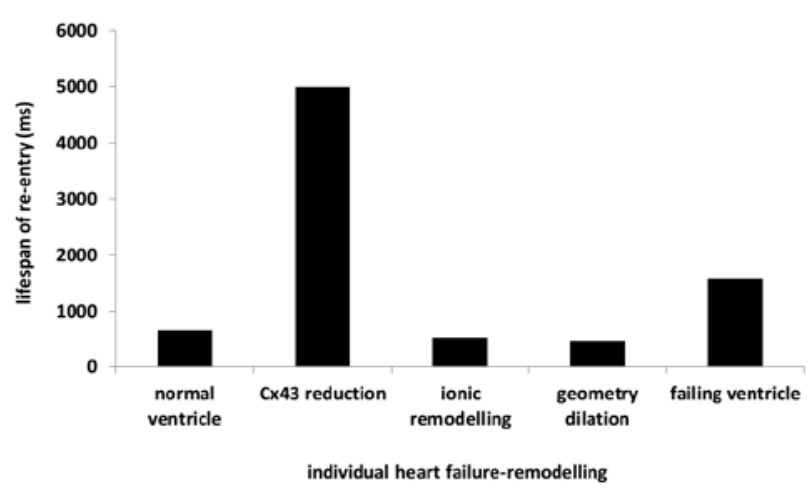

Figure 3. Bar chart of the lifespan of re-entry in the normal ventricle, the ventricle with $\mathrm{Cx} 43$ remodeling, the ventricle with ion channel remodeling, the ventricle with geometry remodeling and the failing ventricle.

\section{Results}

\subsection{Single cell simulations}

In the HF condition, the simulated APs present a similar morphology compared to the CTL condition. However, the characteristics of the APs are altered (Figure 2). The overshoot (OS) and the AP amplitude (APA) are dramatically decreased, along with reduction of the maximum upstroke velocity in the ENDO (by 21.2\%), M (by 20.9\%) and EPI (by 20.9\%), and followed by a less prominent phase 1 repolarisation. Most importantly, APDs are significantly prolonged in the ENDO (by 25.6\%), M (by 55.9\%) and EPI (by 27.1\%). On the other hand, simulation results show a slight shortened APD in PF cells, with increased maximum upstroke velocity under the HF condition.

In all ventricular and the Purkinje fiber cells, the modifications result in a dramatic reduction in the systole $\left[\mathrm{Ca}^{2+}\right]_{\mathrm{i}}$ and an increase in the $\left[\mathrm{Ca}^{2+}\right]_{\mathrm{i}}$ diastole concentration in the HF condition.

These simulated results are in consistence with experimental observations [11, 13-14]. 
3.2. Individual role of heart failureinduced remodeling on sustainability of the ventricular arrhythmias

In this section, role of each individual HF-induced electrical and structural remodeling on lifespan of reentry is investigated. Evolution of the re-entry mapped in Figure 4 and corresponding lifespan of the re-entry is shown in Figure 3.

The initiated re-entry lasted for $620 \mathrm{~ms}$ in control condition and $1500 \mathrm{~ms}$ in HF condition. When the HFreduced conductivity alone was considered, the re-entry lasted for the whole period of simulation of $5000 \mathrm{~ms}$, whilst either ion channel or anatomical remodeling did not alter the sustainability of re-entry compared to control condition.

\subsection{Individual role of revising each heart failure-induced remodeling on sustainability of the ventricular arrhythmias}

In this section, role of individually revising HFinduced remodeling back to normal condition is investigated on lifespan of re-entry initialized by the phase distribution method (shown in Figure 5).

In the absence of intercellular conductivity or geometry remodeling, the re-entry broke up and only lasted for around 500ms in HF condition. However, in absence of ion channel remodeling, the re-entry sustained for more than $5000 \mathrm{~ms}$.

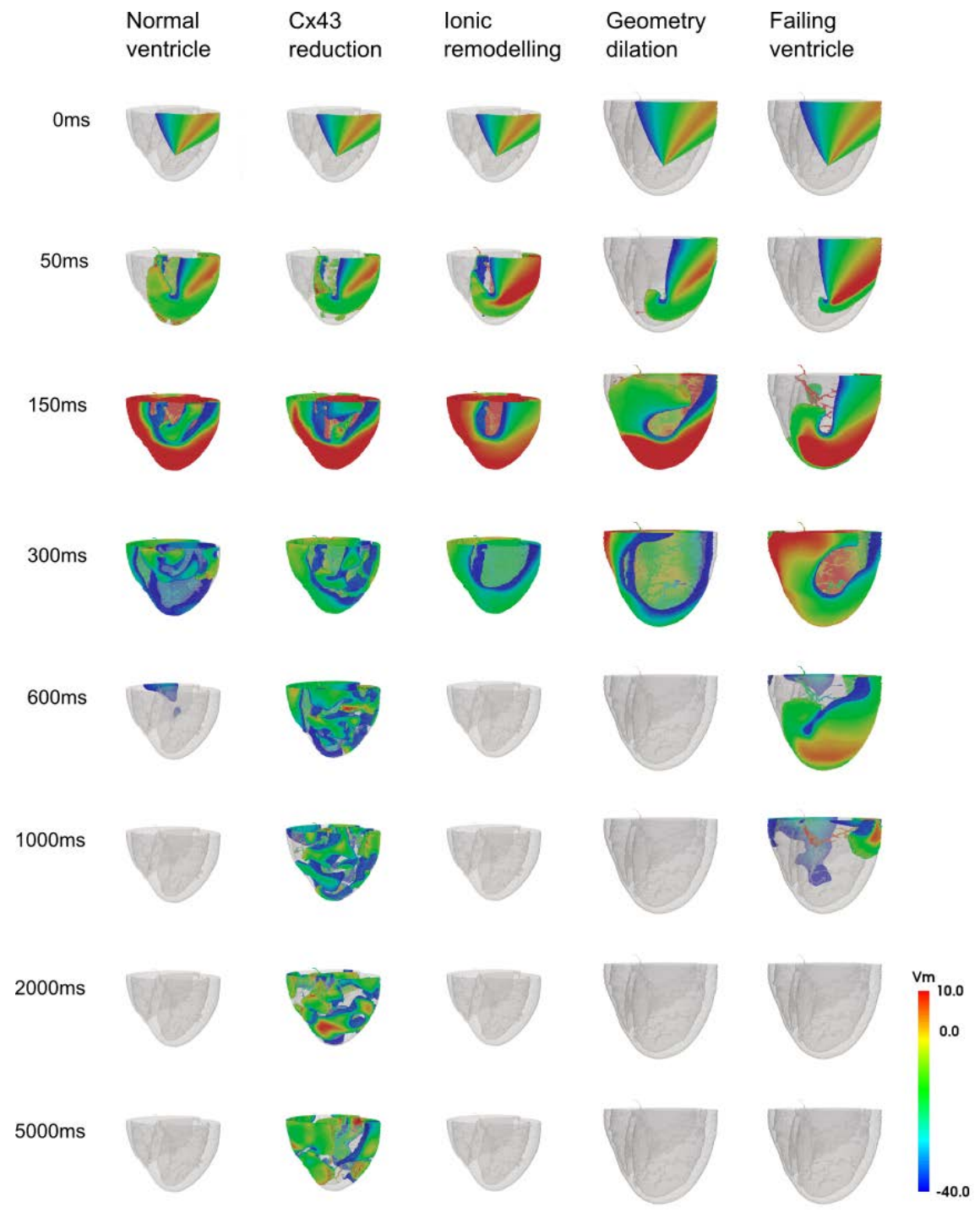

Figure 4. Snapshot of the evolution of re-entry in the normal ventricle, the ventricle with Cx43 remodeling, the ventricle with ion channel remodeling, the ventricle with geometry remodeling and the failing ventricle. 


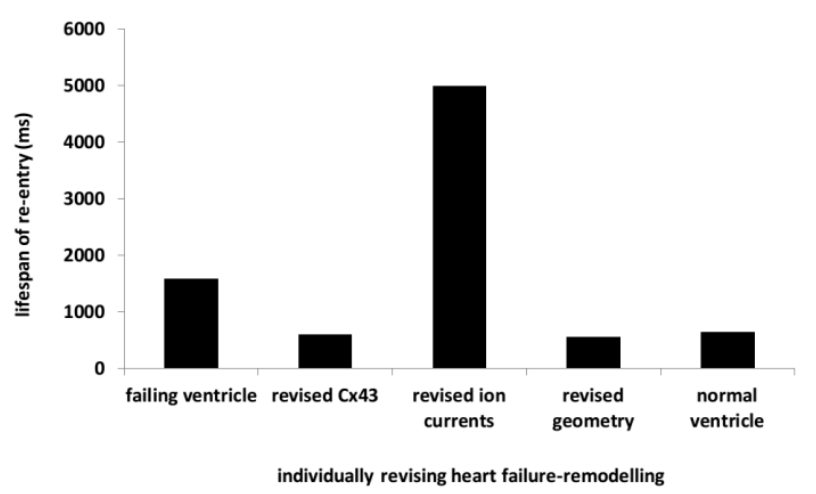

Figure 5. Bar chart of the lifespan of re-entry in the failing ventricle, the ventricle with $\mathrm{Cx} 43$ revised, the ventricle with ion channel revised, the ventricle with geometry revised and the normal ventricle.

\section{Conclusion}

Reduced intercellular coupling increases the excitable gap between the repolarising tail and depolarizing head of successive excitation waves, thus increases sustainability of the re-entry.

APD prolongation is a key factor that is responsible for genesis of ventricular arrhythmias. However, prolongation of APD increases wavelength of the electrical conduction thus reduces sustainability of reentry.

\section{References}

[1] A. Mosterd and A. W. Hoes, 'Clinical epidemiology of heart failure’, Heart, vol. 93, no. 9, pp. 1137-1146, Sep. 2007.

[2] D. Mozaffarian, E. J. Benjamin, A. S. Go, D. K. Arnett, M. J. Blaha, M. Cushman, S. de Ferranti, J.-P. Després, H. J. Fullerton, V. J. Howard, M. D. Huffman, S. E. Judd, B. M. Kissela, D. T. Lackland, J. H. Lichtman, L. D. Lisabeth, S. Liu, R. H. Mackey, D. B. Matchar, D. K. McGuire, E. R. Mohler, C. S. Moy, P. Muntner, M. E. Mussolino, K. Nasir, R. W. Neumar, G. Nichol, L. Palaniappan, D. K. Pandey, M. J. Reeves, C. J. Rodriguez, P. D. Sorlie, J. Stein, A. Towfighi, T. N. Turan, S. S. Virani, J. Z. Willey, D. Woo, R. W. Yeh, and M. B. Turner, 'Heart Disease and Stroke Statistics2015 Update', Circulation, vol. 131, no. 4, pp. e29-e322, Jan. 2015.

[3] D. Levy, S. Kenchaiah, M. G. Larson, E. J. Benjamin, M. J. Kupka, K. K. L. Ho, J. M. Murabito, and R. S. Vasan, 'Long-term trends in the incidence of and survival with heart failure', N. Engl. J. Med., vol. 347, no. 18, pp. 1397-1402, Oct. 2002.

[4] V. L. Roger, S. A. Weston, M. M. Redfield, J. P. Hellermann-Homan, J. Killian, B. P. Yawn, and S. J. Jacobsen, 'Trends in heart failure incidence and survival in a community-based population', JAMA, vol. 292, no.
3, pp. 344-350, Jul. 2004.

[5] A. P. Benson, O. V. Aslanidi, H. Zhang, and A. V. Holden, 'The canine virtual ventricular wall: a platform for dissecting pharmacological effects on propagation and arrhythmogenesis', Prog. Biophys. Mol. Biol., vol. 96, no. 1-3, pp. 187-208, Apr. 2008.

[6] O. V. Aslanidi, P. Stewart, M. R. Boyett, and H. Zhang, 'Optimal velocity and safety of discontinuous conduction through the heterogeneous Purkinje-ventricular junction’, Biophys. J., vol. 97, no. 1, pp. 20-39, Jul. 2009.

[7] B. O’Rourke, D. A. Kass, G. F. Tomaselli, S. Kääb, R. Tunin, and E. Marbán, 'Mechanisms of altered excitation-contraction coupling in canine tachycardiainduced heart failure, I: experimental studies', Circ. Res., vol. 84, no. 5, pp. 562-570, Mar. 1999.

[8] 'The CardioVascular Research Grid'. [Online]. Available: http://cvrgrid.org/. [Accessed: 01-Aug-2016].

[9] P. A. Helm, L. Younes, M. F. Beg, D. B. Ennis, C. Leclercq, O. P. Faris, E. McVeigh, D. Kass, M. I. Miller, and R. L. Winslow, 'Evidence of structural remodeling in the dyssynchronous failing heart', Circ. Res., vol. 98, no. 1, pp. 125-132, Jan. 2006.

[10] P. A. Helm, H.-J. Tseng, L. Younes, E. R. McVeigh, and R. L. Winslow, 'Ex Vivo 3D Diffusion Tensor Imaging and Quantification of Cardiac Laminar Structure', Magn Reson Med, vol. 54, no. 4, pp. 850-859, Oct. 2005.

[11] E. Drouin, F. Charpentier, C. Gauthier, K. Laurent, and H. Le Marec, 'Electrophysiologic characteristics of cells spanning the left ventricular wall of human heart: evidence for presence of M cells', J. Am. Coll. Cardiol., vol. 26, no. 1, pp. 185-192, Jul. 1995.

[12] K. H. W. J. T. Tusscher and A. V. Panfilov, 'Modelling of the ventricular conduction system', Progress in Biophysics and Molecular Biology, vol. 96, no. 1-3, pp. 152-170, Jan. 2008.

[13] F. G. Akar, R. D. Nass, S. Hahn, E. Cingolani, M. Shah, G. G. Hesketh, D. DiSilvestre, R. S. Tunin, D. A. Kass, and G. F. Tomaselli, 'Dynamic changes in conduction velocity and gap junction properties during development of pacing-induced heart failure', American Journal of Physiology - Heart and Circulatory Physiology, vol. 293, no. 2, pp. H1223-H1230, Aug. 2007.

[14] A. I. Undrovinas, V. A. Maltsev, and H. N. Sabbah, 'Repolarization abnormalities in cardiomyocytes of dogs with chronic heart failure: role of sustained inward current', Cell. Mol. Life Sci., vol. 55, no. 3, pp. 494-505, Mar. 1999.

[15] W. Han, D. Chartier, D. Li, and S. Nattel, 'Ionic Remodeling of Cardiac Purkinje Cells by Congestive Heart Failure', Circulation, vol. 104, no. 17, pp. 20952100, Oct. 2001.

Address for correspondence.

Kun Jian

Biological Physics Group, School of Physics and Astronomy University of Manchester, Oxford Road, Manchester M13 9PL. kun.jian@manchester.ac.uk 